\title{
Comunidade de Syrphidae (Diptera): diversidade e preferências florais no Cinturão Verde (Santa Cruz do Sul, RS, Brasil)
}

\author{
Mírian Nunes Morales ${ }^{1} \&$ Andreas Köhler²
}

${ }^{1}$ Departamento de Zoologia, Programa de pós-Graduação em Entomologia, UFPR, Caixa Postal 19020, 81531-980 Curitiba-PR, Brasil. mirian_nm@yahoo.com.br

${ }^{2}$ Laboratório de Entomologia, Departamento de Biologia, UNISC, Av. Independência, 2293, 96815-900 Santa Cruz do Sul-RS, Brasil. andreas@unisc.br

\begin{abstract}
Syrphidae (Diptera) community: diversity and floral preferences in the Green Belt (Santa Cruz do Sul, RS, Brazil). The aim of this study was to investigate the Syrphidae community in the area of the Green Belt of Santa Cruz do Sul, RS, Brazil, as well as to obtain information on the structure and composition of this community, its floral preferences and the interactions between the species in the food resource utilization. The specimens were collected with entomological net, from September/2001 to January/2005. A total of 1.283 syrphid specimens were collected, totaling 88 species distributed in 21 genera. Eristalinae presented the highest number of collected species, followed by Syrphinae and Microdontinae. The genus Palpada Macquart, 1834 and the species P. urotaenia (Curran) were the most abundant. 1.187 syrphids (74 species) were flower visitors of 51 plant species from 23 families, of which Apiaceae and Asteraceae presented the highest number of visitors. The feeding specialization degree varied according to the visited blooming types. The relative easy access to the floral resources with lighter coloration were the main factors regarding the syrphid attraction in the families Apiaceae, Asteraceae, Lauraceae, Malvaceae, Meliaceae, Oleaceae, Poaceae and Solanaceae. The diversity of the syrphid community is regulated by local interactions between the species, mainly between the environmental conditions and the food resources offering.
\end{abstract}

KEYWORDS. Asteraceae; flower-visiting; insect-plant interaction; Palpada.

RESUMO. Comunidade de Syrphidae (Diptera): diversidade e preferências florais no Cinturão Verde. Objetivou-se investigar a comunidade de Syrphidae, do Cinturão Verde de Santa Cruz do Sul, RS, Brasil, permitindo a obtenção de informações acerca da composição e estrutura desta comunidade, suas preferências florais e interações entre as espécies na utilização de recursos alimentares. Realizaram-se coletas com rede entomológica, entre setembro/2001 a janeiro/ 2005. Foram capturados 1.283 espécimes de Syrphidae, representados por 88 espécies, distribuídos em 21 gêneros. Eristalinae apresentou o maior número de espécies coletadas, seguida por Syrphinae e Microdontinae. O gênero Palpada Macquart e a espécie P. urotaenia (Curran) foram os mais abundantes. As coletas alcançaram cerca de $80 \%$ do que se estima para a área de estudo. Coletaram-se 1.187 sirfídeos (74 espécies) visitantes de 51 espécies de plantas, de 23 famílias, onde Apiaceae e Asteraceae apresentaram o maior número de visitantes. O grau de especialização alimentar variou de acordo com os tipos de flores visitadas. A facilidade do acesso aos recursos florais e a coloração clara das flores são os principais responsáveis pela atração dos sirfídeos em Apiaceae, Asteraceae, Lauraceae, Malvaceae, Meliaceae, Oleaceae, Poaceae e Solanaceae. A diversidade da comunidade de Syrphidae é regulada por interações locais entre as espécies, principalmente entre as condições ambientais e disponibilidade de recursos alimentares.

PALAVRAS-CHAVE. Asteraceae; interação inseto-planta; Palpada; visitantes florais.

Conforme Thompson (1999) a região Neotropical é rica em espécies de Syrphidae, sendo que provavelmente apenas a metade do número real é conhecida. A família Syrphidae está dividida em três subfamílias: Eristalinae, Syrphinae e Microdontinae, a última com distribuição primordialmente Neártica e as duas primeiras cosmopolitas (Vockeroth \& Thompson 1987). Eristalinae possui mais de 900 espécies neotropicais, Syrphinae cerca de 700 e Microdontinae mais de 400 (Thompson 1999, 2005; Marinoni \& Thompson 2003).

Estima-se que para todo o Brasil possam existir cerca de 2.030 espécies de Syrphidae, das quais, aproximadamente, 1.500 ocorreriam no Sul do Brasil (Thompson et al. 1976; Marinoni \& Thompson 2003). São conhecidos 60 gêneros de Syrphidae neotropicais, dentre os quais destacam-se pelo grande número de espécies: Copestylum Macquart, 1846, com aproximadamente 400 espécies; Ocyptamus Macquart, 1834, com aproximadamente 300; Microdon Meigen, 1803 com cerca de 170; Toxomerus Macquart, 1855, com 145; e Palpada Macquart, 1834 com 85 espécies neotropicais (Thompson 1981, 1999, 2003; Marinoni et al. 2004; Rotheray et al. 2007).

Atualmente existe uma grande dificuldade na identificação das espécies de Syrphidae da região Neotropical, devido à falta de chaves taxonômicas e à necessidade de revisões taxonômicas, principalmente para os grupos com muitas espécies, como os citados acima (Marinoni et al. 2004).

Devido à sua importância como polinizadores e no controle de diversos afídeos, este grupo de moscas-das-flores deve ser estudado em seus mais diversos aspectos. Para que estudos aplicados possam ser futuramente realizados, tornase necessário o desenvolvimento de estudos relacionados à biodiversidade destes organismos em uma determinada região. De acordo com Ricklefs (1996), a biodiversidade reflete um amplo conjunto de processos locais, regionais e históricos, e eventos que operam em uma hierarquia de escalas espaciais e 
temporais. Deste modo, a compreensão dos padrões de diversidade das espécies requer a consideração da história da região e a interação, principalmente, com estudos ecológicos, de sistemática, bionomia, evolução e biogeografia.

Portanto, o presente estudo objetivou investigar a comunidade de Syrphidae, na área do Cinturão Verde de Santa Cruz do Sul, Rio Grande do Sul, Brasil, permitindo a obtenção de informações acerca da composição e estrutura desta comunidade, suas preferências florais e interações entre as espécies na utilização de recursos alimentares.

\section{MATERIALE MÉTODOS}

Área de estudo. A área de estudo compreendeu o Cinturão Verde, localizado na área urbana do município de Santa Cruz do Sul. Conforme Decreto no 4117 de 1994, é uma área de 465 hectares de vegetação secundária, com perímetro linear de 32 Km (Alves \& Colischonn 2001). Atualmente, as áreas privadas do Cinturão Verde estão sendo desmatadas para a construção civil e agricultura.

A altitude varia entre 50 e 150 metros acima do nível do mar, o clima é classificado como úmido, com temperaturas médias inferiores a $15^{\circ} \mathrm{C}$ nos meses de inverno e superiores a $20^{\circ} \mathrm{C}$ nos meses de verão (Alves \& Colischonn 2001).

Métodos de coleta, identificação e análise dos dados. As coletas foram realizadas com rede entomológica na vegetação em floração existente na borda da mata, pelo menos uma vez por semana, quando o tempo era favorável (ensolarado e sem chuva), entre as 8-17 horas, no período entre setembro de 2001 e janeiro de 2005.

Escolheram-se aleatoriamente plantas floridas e após a observação da presença de insetos visitantes, realizaram-se batidas durante 10 minutos em cada planta. A área de coleta foi dividida em duas partes: Norte e Sul. A cada dia as coletas se iniciavam no sentido oposto da primeira, ou seja, o primeiro dia de coleta se iniciou pela manhã na parte norte e à tarde na parte sul, o dia seguinte se iniciou pela manhã na parte sul e à tarde na parte norte, e assim sucessivamente.

Os insetos foram colocados com câmaras mortíferas com acetato de etila e separados em intervalos de uma hora. A cada hora verificou-se a temperatura do local com termômetro digital.

Os insetos coletados foram analisados no Laboratório de Entomologia da Universidade de Santa Cruz do Sul (UNISC) e depositados na sua Coleção Entomológica. Os sirfídeos foram identificados com auxílio de chaves dicotômicas ou por comparação com o material da Coleção de Entomologia Pe. J. S. Moure, Departamento de Zoologia, Universidade Federal do Paraná e do Museu Nacional do Rio de Janeiro.

De todas as plantas com registro de visitação foi feita uma exsicata para posterior identificação no Herbário da UNISC.

Para a análise dos dados o período de coleta foi dividido em 14 amostragens, ordenadas de "A" a "N", organizadas por trimestre, iniciando-se em setembro de 2001.

A freqüência absoluta dos sirfídeos foi determinada mediante a quantificação do total de espécimes coletados de cada espécie diferente.
Para os cálculos de abundância e dominância dos gêneros e espécies, utilizou-se o índice de dominância e abundância proposto por Lobo \& Leighton (1986).

A riqueza das espécies de Syrphidae foi estimada através do programa Statistical estimation of species richness and shared species from samples, Version 6.0 bl (Colwell 2000).

Verificou-se a atividade dos sirfídeos em relação à temperatura através da quantificação dos espécimes nas capturas em intervalos de $1^{\circ} \mathrm{C}$. Utilizou-se o teste "U" de MannWhitney para verificar a preferência pelas temperaturas.

\section{RESULTADOS}

No período de setembro de 2001 a janeiro de 2005 foram capturados 1.283 espécimes de Syrphidae, representando 88 espécies, distribuídas em 21 gêneros (Tab. I).

Abundância e riqueza de espécies. A subfamília Eristalinae apresentou o maior número de espécies coletadas (46 espécies), seguida por Syrphinae e Microdontinae com 37 e 5 espécies respectivamente. Do total de espécies de Syrphidae, 14 não foram coletadas visitando flores (Tab. I).

O gênero mais abundante e de maior riqueza foi Palpada, com 677 indivíduos e 17 espécies, seguido por Toxomerus com 235 indivíduos e 16 espécies. O terceiro e quarto gêneros com maior riqueza foram Copestylum e Ocyptamus, ambos com 12 espécies coletadas. $\mathrm{O}$ terceiro e quarto gêneros mais abundantes foram Ornidia e Allograpta, com 161 e 51 espécimes coletados respectivamente (Tab. I).

Analisando-se a estrutura temporal da comunidade de Syrphidae verificou-se o maior número de indivíduos coletados nas épocas de primavera e verão (A, B, E, F e I) sendo que as amostras E e F representaram juntas 52,22\% dos espécimes coletados (Fig. 1).

Neste contexto, observou-se uma forte redução das coletas a partir da amostragem $\mathrm{J}$, devido às condições climáticas desfavoráveis do período juntamente com o manejo da vegetação realizado pelos proprietários da área de estudo.

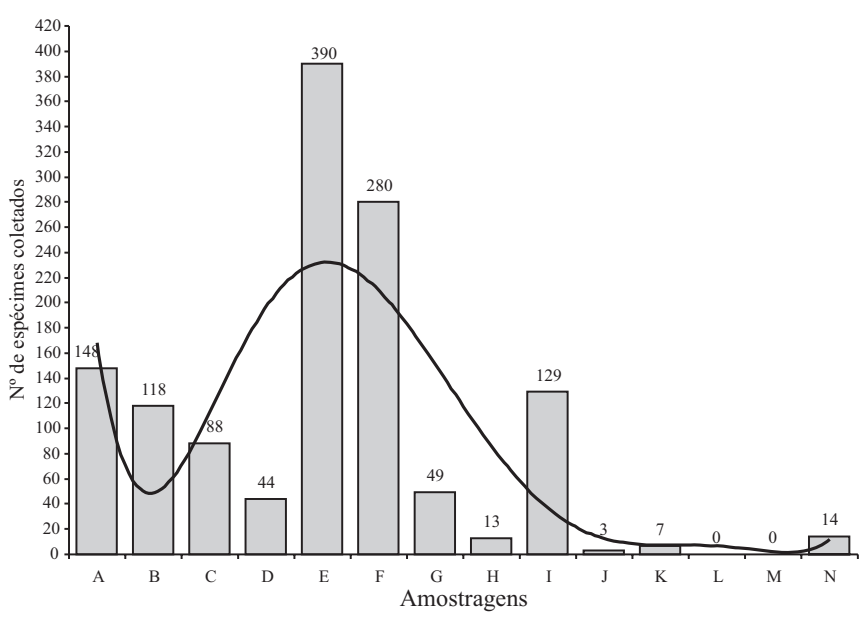

Fig. 1. Número de espécimes de Syrphidae coletados, por trimestre, de Setembro de 2001 a Janeiro de 2005, ao longo das amostragens, na área do Cinturão Verde de Santa Cruz do Sul, RS. Linha de tendência polinomial de sexta ordem. 


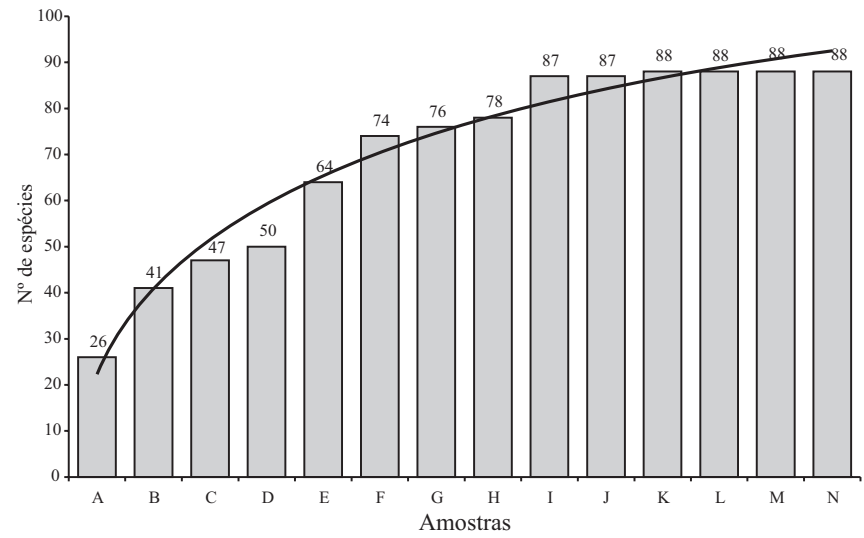

Fig. 2. Histograma do acúmulo da ocorrência das espécies de Syrphidae, coletados por trimestre, de Setembro de 2001 a Janeiro de 2005, na área do Cinturão Verde, Santa Cruz do Sul, RS, setembro/2001 a janeiro/ 2005. Linha de tendência logarítmica.

Os estimadores da riqueza de espécies (Cowell 2000) indicaram a possibilidade de ocorrer na área, de 110 a 150 espécies de Syrphidae. Utilizando-se o valor médio dos estimadores, verifica-se que o número de espécies de Syrphidae coletado até o momento está entre $60 \%$ e $80 \%$ do que realmente possa ocorrer na área em questão.

A Figura 2 apresenta o acúmulo da ocorrência de novas espécies de Syrphidae ao longo das amostragens. A linha de tendência demonstra que o número de espécies tende a aumentar, o que é corroborado com os estimadores acima citados. Os meses de primavera e verão apresentaram um maior aumento de novas ocorrências, sendo que na primavera este número é mais elevado, diminuindo gradativamente no verão, outono e inverno.

Sirfídeos visitantes de flores. Foram coletados 1.187 sirfídeos (74 espécies) visitantes de 51 espécies de plantas, distribuídas em 23 diferentes famílias (Tabs. II e III).

As famílias Apiaceae e Asteraceae apresentaram o maior número de espécies e de espécimes de Syrphidae visitantes. Juntas, representaram $75,06 \%$ do total de indivíduos coletados em flores. As demais famílias apresentaram entre 4,04\% a 0,08\% do total de visitantes (Tab. III).

Foram coletados sirfídeos em apenas duas espécies da família Apiaceae: Eryngium horridum Malme e Eryngium megapotamicum Malme. Nestas, obtiveram-se 572 sirfídeos visitantes de flores, distribuídos em 56 espécies. Na família Asteraceae, foram coletados sirfídeos visitando as flores de 14 espécies: Baccharis trimera (Less.); Baccharis sp. 1; Cosmos bipinnatus Cav.; Elephantopus mollis Kunth; Eupatorium laevigatum Lam.; Senecio brasiliensis (Spreng.) Less; Vernonia florida Gardn; Vernonia oxyodonta Malme; Vernonia polyanthes Less.; Vernonia puberula Less.; sp. 1; sp. 2; sp. 3 e sp. 4 . No entanto, apesar do maior número de espécies de asteráceas, foram coletados menos sirfídeos visitantes em relação ao número coletado nas apiáceas: 319 sirfídeos, distribuídos em 35 espécies (Tabs. II e III).

Avaliando-se a relação entre o número de espécimes e de espécies visitantes por família de planta (Fig. 3), verificou-se que Apiaceae e Asteraceae foram as famílias mais importantes na atividade forrageadora da comunidade de Syrphidae amostrada no período.

Neste sentido, em um nível intermediário, outras sete famílias de plantas destacaram-se na manutenção da atividade dos sirfídeos: Fabaceae, Lauraceae, Malvaceae, Meliaceae, Oleaceae, Poaceae e Solanaceae. O número de espécimes visitantes destas famílias apresentou-se semelhante, entre 22 a 48 indivíduos; no entanto, Malvaceae e Poaceae destacamse das cinco demais famílias por apresentaram uma proporção maior do número de espécies visitantes em relação ao número de indivíduos coletados.

Doze espécies de Syrphidae foram consideradas abundantes nesta comunidade, conforme o índice de dominância e abundância (Lobo \& Leighton 1986): Allograpta exotica (Widemann, 1830), Ornidia obesa (Fabricius, 1775), Palpada agrorum (Fabricius, 1787), Palpada furcata (Wiedemann, 1819), Palpada precipua (Williston, 1888), Palpada pusilla (Macquart, 1842), Palpada urotaenia (Curran, 1930), Pseudodoros clavatus (Fabricius, 1794), Syrphus phaeostigma Wiedemann, 1830, Toxomerus virgulatus (Macquart, 1850), Toxomerus sp. 4 e Toxomerus sp. 5 (Tab. II). É interessante ressaltar que $O$. obesa, $P$. furcata e $P$. urotaenia representaram juntas 47,94\% dos espécimes de Syrphidae visitantes florais.

A ocorrência dos sirfídeos variou conforme a temperatura entre 27 e $35^{\circ} \mathrm{C}$, com maior frequiência no intervalo de 30$30,9^{\circ} \mathrm{C}$ (Tab. IV). Através do teste "U” de Mann-Whitney verificou-se uma preferência pelas temperaturas entre 28 e $32^{\circ} \mathrm{C}$, onde estão incluídos 76,66\% dos indivíduos coletados.

Os horários que apresentaram maior freqüência de sirfídeos

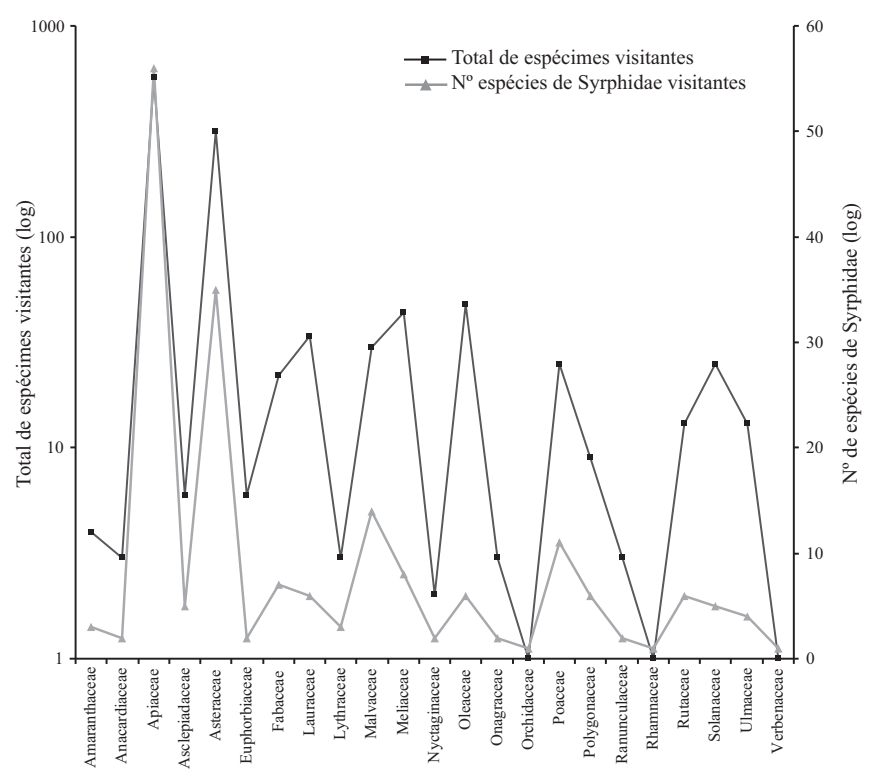

Fig. 3. Relação entre o número de espécimes e de espécies de Syrphidae coletados visitando as flores de 23 famílias de plantas, no Cinturão Verde de Santa Cruz do Sul, RS, no período de setembro/2001 a janeiro/ 2005. 
Tabela I. Espécies da família Syrphidae coletadas no Cinturão Verde de Santa Cruz do Sul, organizadas por Subfamílias, no período de setembro de 2001 a janeiro de 2005 .

\begin{tabular}{|c|c|c|c|}
\hline Subfamília / Espécies & $\begin{array}{c}\text { Número } \\
\text { de } \\
\text { indivíduos }\end{array}$ & Subfamília / Espécies & $\begin{array}{c}\text { Número } \\
\text { de } \\
\text { indivíduos }\end{array}$ \\
\hline
\end{tabular}

Eristalinae (46 espécies)

Ceriana (Sphiximorpha) wulpii (Williston, 1888)

Chalcosyrphus (Neplas) armatipes (Curran, 1941)

Copestylum (Lepidopsis) compactum (Curran, 1925)

Copestylum (Phalacromya) belinda (Hull, 1949)

Copestylum (Phalacromya) circe (Curran, 1939)

Copestylum (Phalacromya) clarum (Hull, 1942)

Copestylum (Phalacromya) flukei (Curran, 1936)

Copestylum (Phalacromya) lanei (Curran, 1936)

Copestylum (Phalacromya) obscurior (Curran, 1939)

Copestylum (Phalacromya) pallens (Wiedemann, 1830)

Copestylum (Phalacromya) spinigerum (Wiedemann,1830) $1 *$

Copestylum sp. 1

Copestylum sp. 2

Copestylum sp. 3

Eristalinus (Eristalodes) taeniops (Wiedemann, 1818)

Eristalis tenax (Linnaeus, 1758)

Lycopale chrysotaenia (Fluke, 1937)

Meromacrus nectarinoides (Lynch Arribálzaga, 1892)

Meromacrus niger Sack, 1920

Ornidia major Curran, 1930

Ornidia obesa (Fabricius, 1775)

Palpada agrorum (Fabricius, 1787)

Palpada distinguenda (Wiedemann,1830)

Palpada doris (Curran, 1930)

Palpada expicta (Walker, 1860)

Palpada furcata (Wiedemann,1819)

Palpada minutalis (Williston, 1891)

Palpada precipua (Williston, 1888)

Palpada pusilla (Macquart, 1842)

Palpada pygolampa (Wiedemann, 1830)

Palpada rufipedes Thompson, 1976

Palpada rufoscutellata (Sack, 1921)

Palpada scutellaris (Fabricius, 1805)

Palpada urotaenia (Curran, 1930)

Palpada vera (Hull, 1949)

Palpada sp. 1

Palpada sp. 2

Palpada sp. 3

Quichuana bezzi Ceresa, 1934

Quichuana picadoi Knab, 1913

Quichuana pogonosa Fluke, 1937

Quichuana sp. 1

Rhingia sp. 1

Sterphus (Crepidomyia) shannoni Thompson, 1973

Syritta flaviventris Macquart, 1842

Trichopsomyia sp. 1
Syrphinae (37 espécies)

Allograpta exotica (Wiedemann, 1830) 26

Allograpta hastata Fluke, 1942

Allograpta neotropica Curran, 1936

Allograpta similis Curran, 1925

Allograpta strigifacies (Enderlein, 1938) 1

Argentinomyia pollinosus (Hull, 1942)

Argentinomyia sp. 1

Ocyptamus (Mimocalla) bonariensis (Brèthes, 1905) $\quad 1^{*}$

Ocyptamus aff. caldus (Walker, 1852) 2*

Ocyptamus antiphates (Walker, 1849) 2

Ocyptamus arabella (Hull, 1947) 1

Ocyptamus clarapex (Wiedemann, 1830) 3

Ocyptamus gastrostactus (Wiedemann, 1830) 1

Ocyptamus laticauda (Curran, 1941) 2

Ocyptamus luctuosus (Bigot, 1884) 1*

Ocyptamus phaeopterus (Schiner, 1868) 2

Ocyptamus stenogaster (Williston, 1888) 3

Ocyptamus sp. 1

Ocyptamus sp. 2

Pseudodoros clavatus (Fabricius, 1794) 38

Syrphus phaeostigma Wiedemann, 1830

Toxomerus basalis (Walker, 1836) 3

Toxomerus corbis (Walker 1852) 1

Toxomerus dispar (Fabricius, 1794) 15

Toxomerus aff. marginatus (Say, 1823) 5

Toxomerus musicus (Fabricius, 1805) 8

Toxomerus pictus (Macquart, 1842) $1^{*}$

Toxomerus politus (Say, 1823) 8

Toxomerus tibicen (Wiedemann, 1830) 1

Toxomerus virgulatus (Macquart,1850) 39

Toxomerus watsoni (Curran, 1930) 13

Toxomerus sp. nov.

Toxomerus sp. 1

Toxomerus sp. 2

Toxomerus sp. 3

Toxomerus sp. $4 \quad 24$

Toxomerus sp. 5

Microdontinae (05 espécies)

Microdon pilosops Marinoni, $2004 \quad 2 *$

Microdon tigrinus Curran, $1940 \quad 2$

Microdon sp. $1 \quad 1$ *

Microdon sp. $2 \quad 1 *$

Microdon sp. $3 \quad 1 *$

Total 88 espécies

1.283

(*) espécimes coletados em vôo sobre a vegetação.

\section{DISCUSSÃO}

capturados foram no intervalo entre as 10 e 14 horas (Fig. 4). A linha de tendência polinomial evidenciou dois picos principais, entre as 10 e 11 horas e às 14 horas, o que esteve diretamente relacionado à ocorrência das temperaturas preferenciais nestes horários.

*




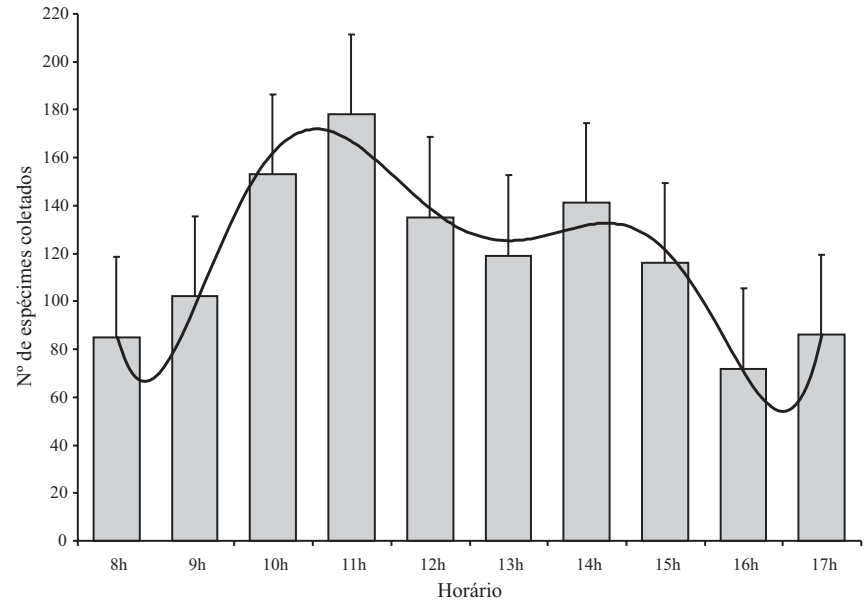

Fig. 4. Número de espécimes de Syrphidae coletados visitando flores conforme horário, no Cinturão Verde de Santa Cruz do Sul, RS, no período de setembro/2001 a janeiro/2005. Linha de tendência polinomial de sexta ordem e desvio padrão.

Syrphinae, como foi observado neste estudo, pois a maioria dos adultos destes grupos costuma dispensar bastante tempo nas atividades forrageadoras. Contudo, as espécies de Microdontinae são raramente coletadas com este tipo de armadilha. No caso, cinco espécies de Microdon foram coletadas e apenas uma foi capturada visitando flores. Este é um gênero cujas espécies usualmente não se alimentam de recursos florais (Duffield 1981). Desta forma, pode-se esperar que em coletas com armadilha de Malaise espécies de Microdon apareçam com maior freqüência, conforme observado em Marinoni et al. (2004) e Marinoni \& Bonatto (2002), pois acabam voando mais por não dispensarem muito tempo alimentando-se nas flores.

Os gêneros mais abundantes e de maior riqueza compreenderam justamente aqueles mais comuns e mais diversos de Syrphidae da região Neotropical (Thompson 1981, 1999, 2003; Marinoni et al. 2004; Rotheray et al. 2007).

A composição da comunidade de Syrphidae pode estar associada tanto aos hábitos das larvas quanto dos adultos (Gilbert et al. 1985; Pascarella et al. 2001). Um pico de espécies em determinada época pode estar não somente relacionado à disponibilidade de alimento para os adultos, mas estar ligado às condições apropriadas para o desenvolvimento dos estágios imaturos.

Os estágios imaturos de Syrphidae são pouco conhecidos, porém, dos gêneros coletados com maior riqueza, sabe-se que os imaturos das espécies de Palpada e Copestylum são saprófagos, desenvolvendo-se comumente em cavidades de troncos de árvores e em plantas que acumulam água formando fitotelmata, como em bromélias (Pérez-Bañon et al. 2003; Rotheray et al. 2007). Na área de estudo, a grande quantidade de Eryngium horridum Malme poderia estar contribuindo para o desenvolvimento dos imaturos destes gêneros, pois a disposição de suas folhas em forma de roseta é propícia ao armazenamento água para a formação de fitotelmata.

Entretanto, os imaturos das espécies de Toxomerus e
Ocyptamus são geralmente encontrados predando afídeos, assim, da mesma forma para as espécies dos gêneros acima citados, estudos acerca da biologia das larvas são necessários para que mais inferências sobre estas interações possam ser realizadas.

No final da primavera de 2003 ocorreu um período de seca, estendendo-se a meados de abril, onde se iniciou o período de frio e chuvas; no final de dezembro de 2003 a área onde crescia Eryngium horridum foi manejada e neste local os proprietários plantaram árvores frutíferas. Novamente, no verão seguinte (2004/2005) ocorreu um forte período de seca e calor intenso, prejudicando a floração das plantas, e que combinados ao manejo da vegetação e provavelmente à falta de condições para o desenvolvimento dos imaturos, contribuíram para a redução da ocorrência dos sirfídeos na área de estudo.

A área amostrada está sendo constantemente manejada e recentemente utilizada para a construção civil, portanto as estimativas de que ainda faltam pouco mais de $20 \%$ de espécies de Syrphidae a serem reconhecidas na área de estudo, podem se tornar nulas com o passar dos anos, pois como foi observado a partir da amostragem $\mathbf{J}$ (Fig. 1), a redução do número de espécimes coletados é bastante grande, embora com o efeito de condições climáticas adversas.

Sirfídeos visitantes de flores. Diversos estudos já indicaram que a família Asteraceae compreende o maior número de espécies com registro de visitação por sirfídeos, e Apiaceae apresenta os dípteros como os visitantes florais mais freqüentes, o que corrobora com as observações deste trabalho (Arruda 1997; Arruda et al. 1998; Proctor et al. 1996; Morales \& Köhler 2006).

Dos gêneros de Syrphidae mais abundantes coletados visitando flores, em outros estudos realizados no Brasil, Palpada também compreendeu o gênero mais abundante; da mesma forma, $P$. furcata e Ornidia obesa aparecem entre as espécies de Syrphidae mais freqüentes (Souza-Silva et al. 2001; Arruda 1997; Arruda et al. 1998; Machado \& Loiola 2000; Morales \& Köhler 2006).

Conforme Arruda (1997), P. furcata provavelmente ocupa uma posição elevada nas relações de dominância entre as espécies de Syrphidae.

O predomínio de sirfídeos robustos e mais ágeis sobre sirfídeos de menor porte como Toxomerus e Allograpta, pode ser resultante de uma competição interespecífica (Morales \& Köhler 2006). Contudo, o formato da probóscide e do labelo das espécies visitantes podem ser um fator importante no seu hábito forrageador. Gilbert (1981) observou que as espécies de Syrphidae que se alimentam de pólen possuem probóscide curta e labelo largo; espécies que se alimentam de néctar têm probóscidae longa e labelo relativamente curto, com presença de algumas cerdas, enquanto as de hábito alimentar misto possuem formas intermediárias ou se parecem às que se alimentam de néctar.

As espécies de maior tamanho normalmente são as que se alimentam de néctar, provavelmente por necessitarem de mais energia do que as de menor tamanho. Isto pode corroborar o alto índice de sirfídeos visitantes em Apiaceae. 
Tabela II. Número de espécimes e freqüências absoluta e relativa das espécies de Syrphidae visitantes das flores de 23 famílias de plantas, na área do Cinturão Verde de Santa Cruz do Sul, RS, no período de setembro de 2001 a janeiro de 2005.

Famílias de plantas

\begin{tabular}{|c|c|c|c|c|c|c|c|c|c|c|c|c|c|c|c|c|c|c|c|c|c|c|c|c|c|}
\hline Espécies de Syrphidae & 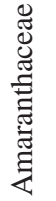 & 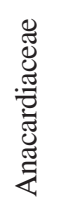 & $\begin{array}{l}\mathbb{E} \\
\stackrel{0}{0} \\
\frac{\pi}{2} \\
\frac{\pi}{4}\end{array}$ & 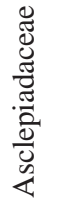 & 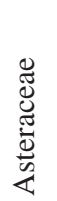 & 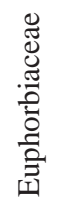 & 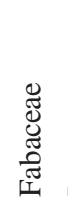 & 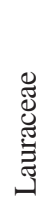 & 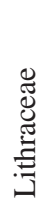 & 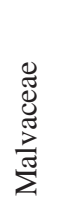 & 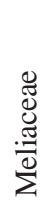 & 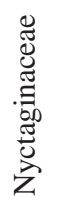 & 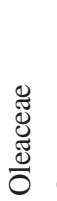 & 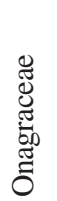 & 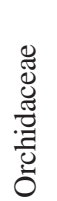 & 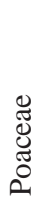 & 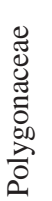 & 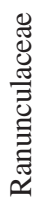 & 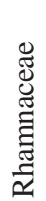 & 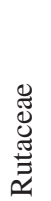 & 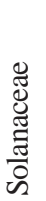 & 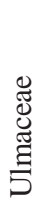 & 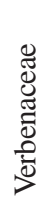 & $\begin{array}{c}\text { Freq. } \\
\text { absoluta }\end{array}$ & $\begin{array}{c}\text { Freq. } \\
\text { relativa } \\
{[\%]}\end{array}$ \\
\hline
\end{tabular}

Allograpta exotica*

Allograpta hastata

Allograpta neotropica

Allograpta similis

Allograpta strigifacies

Argentinomyia pollinosa

Argentinomyia sp. 1

Ceriana wulpii

Chalcosyrphus armatipes

Copestylum belinda

Copestylum circe

Copestylum clarum

Copestylum compactum

Copestylum flukei

Copestylum lanei

Copestylum obscurior

Copestylum pallens

Copestylum sp. 1

Eristalinus taeniops

Eristalis tenax

Lycopale chrysotaenia

Meromacrus niger

Microdon tigrinus

Ocyptamus anthiphates

Ocyptamus arabela

Ocyptamus clarapex

Ocyptamus gastrostactus

Ocyptamus laticauda

Ocyptamus phaeopterus

Ocyptamus stenogaster

Ocyptamus sp. 1

Ornidia major

Ornidia obesa *

Palpada agrorum *

Palpada distinguenda

Palpada doris

Palpada expicta

Palpada furcata*

Palpada minutalis

Palpada precipua*

Palpada pusilla*

Palpada pygolampa

Palpada rufipedes

Palpada rufoscutellata

Palpada scutellaris

Palpada urotaenia*

Palpada vera

Palpada sp. 1

Palpada sp. 2

Palpada sp. 3

Pseudodoros clavatus *

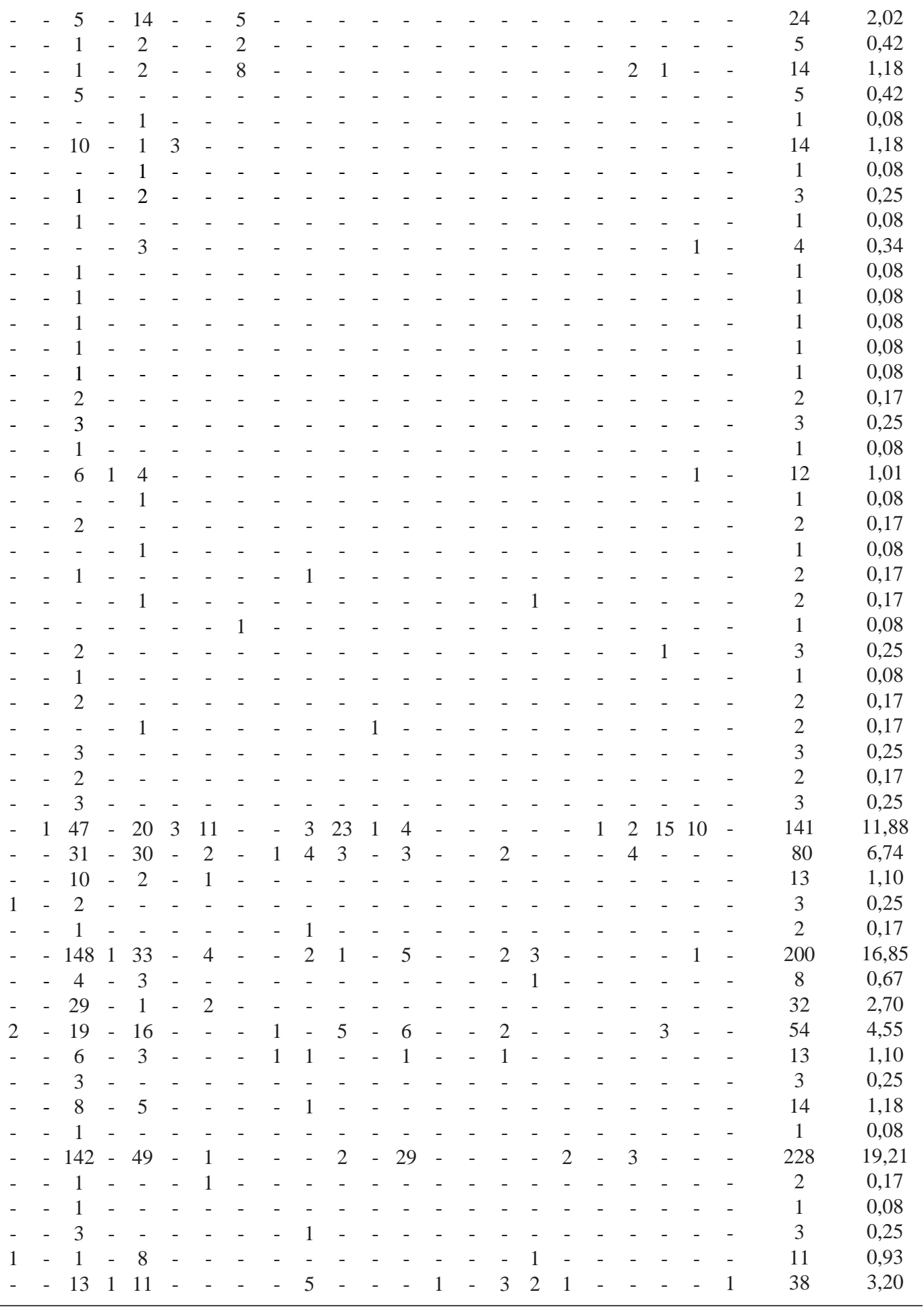


Tabela II. Continuação.

Famílias de plantas

\begin{tabular}{|c|c|c|c|c|c|c|c|c|c|c|c|c|c|c|c|c|c|c|c|c|c|c|c|}
\hline Espécies de Syrphidae & 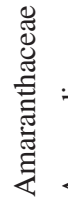 & 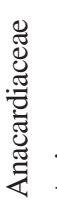 & 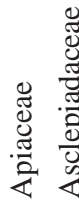 & 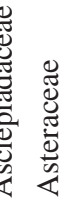 & 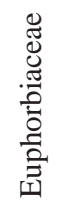 & 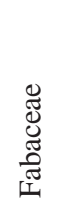 & 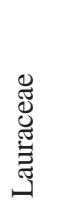 & 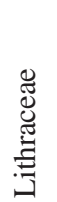 & 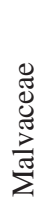 & 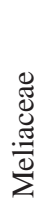 & 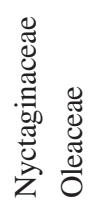 & 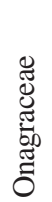 & 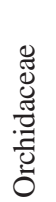 & 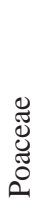 & 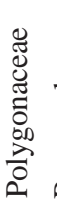 & 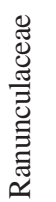 & 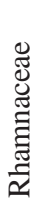 & 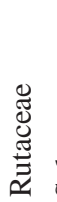 & 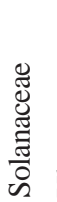 & 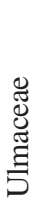 & 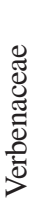 & $\begin{array}{c}\text { Freq. } \\
\text { absoluta }\end{array}$ & $\begin{array}{c}\text { Freq. } \\
\text { relativa } \\
{[\%]}\end{array}$ \\
\hline
\end{tabular}

Quichuana bezzii

Quichuana picadoi

Quichuana pogonosa

Quichuana sp. 1

Sterphus shannoni

Syritta flaviventris

Syrphus phaeostigma *

Toxomerus basalis

Toxomerus corbis

Toxomerus dispar

Toxomerus aff. marginatus

Toxomerus musicus

Toxomerus politus

Toxomerus tibicen

Toxomerus virgulatus *

Toxomerus watsoni

Toxomerus sp. 1

Toxomerus sp. 2

Toxomerus sp. 3

Toxomerus sp. 4 *

Toxomerus sp. $5 *$

Toxomerus sp. nov.

Trichopsomyia sp. 1

\begin{tabular}{|c|c|c|c|c|c|c|c|c|c|c|c|c|c|c|c|c|c|c|c|c|c|c|c|}
\hline & -1 & - & - & - & - & - & - & - & - & - & - & - & 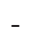 & - & - & - & 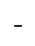 & - & - & - & - & 1 & 0,08 \\
\hline & -1 & - & - & - & - & - & - & - & - & - & - & - & - & - & - & - & - & - & - & - & . & 1 & 0,08 \\
\hline & $-\quad-$ & - & 1 & - & - & - & - & - & - & - & - & - & - & - & - & - & - & - & - & - & - & 1 & 0,08 \\
\hline & -1 & - & - & - & - & - & - & - & - & - & - & - & - & - & - & - & - & - & - & - & - & 1 & 0,08 \\
\hline & -1 & - & 3 & - & - & - & - & - & - & - & - & - & - & - & - & - & - & - & - & - & 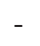 & 4 & 0,34 \\
\hline & - 1 & - & - & - & - & - & - & - & - & - & - & - & - & - & - & - & - & - & - & - & - & 1 & 0,08 \\
\hline & $-\quad-$ & - & - & - & - & 16 & - & 1 & - & - & - & - & - & - & - & - & - & 1 & - & - & - & 18 & 1,52 \\
\hline & $-\quad-$ & - & - & - & - & - & - & 3 & - & - & - & - & - & - & - & - & - & - & - & - & - & 3 & 0,25 \\
\hline & $-\quad-$ & - & - & - & - & - & - & 1 & - & - & - & - & - & - & - & - & - & - & - & - & - & 1 & 0,08 \\
\hline . & $-\quad-$ & 2 & 3 & - & - & - & - & 1 & 6 & - & - & - & - & 2 & 1 & - & - & - & - & - & - & 15 & 1,26 \\
\hline - & -2 & 1 & - & - & - & - & - & - & 2 & - & - & - & - & - & - & - & - & - & - & - & - & 5 & 0,42 \\
\hline- & - 1 & - & - & - & - & - & - & - & - & - & - & - & - & 4 & - & - & - & - & - & - & - & 5 & 0,42 \\
\hline 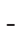 & $-\quad-$ & - & 1 & - & - & 2 & - & - & - & - & - & - & - & 4 & - & - & - & 1 & - & - & - & 8 & 0,67 \\
\hline- & $-\quad-$ & - & 1 & - & - & - & - & - & - & - & - & - & - & - & - & - & - & - & - & - & - & 1 & 0,08 \\
\hline- & 24 & - & 23 & - & - & - & - & 4 & - & - & - & - & 1 & - & - & - & - & - & - & - & - & 34 & 2,86 \\
\hline- & - 1 & - & 7 & - & - & - & - & 2 & - & - & - & - & - & 2 & - & - & - & - & - & - & - & 12 & 1,01 \\
\hline- & -2 & - & - & - & - & - & - & - & - & - & - & - & - & 2 & - & - & - & - & - & - & - & 4 & 0,34 \\
\hline- & -3 & - & 1 & - & - & - & - & - & - & - & - & - & - & - & - & - & - & - & 5 & - & - & 9 & 0,76 \\
\hline - & -3 & - & - & - & - & - & - & - & - & - & - & - & - & - & - & - & - & - & - & - & - & 3 & 0,25 \\
\hline - & -24 & - & - & - & - & - & - & - & - & - & - & - & - & - & - & - & - & - & - & - & - & 24 & 2,02 \\
\hline - & $-\quad-$ & - & 63 & - & - & - & - & - & 2 & - & - & 2 & - & - & - & - & - & - & - & - & - & 67 & 5,64 \\
\hline - & - & - & - & - & - & - & - & - & - & - & - & - & - & 1 & - & - & - & - & - & - & - & 1 & 0,08 \\
\hline- & $-\quad-$ & - & 1 & - & - & - & - & - & - & - & - & - & - & - & - & - & - & - & - & - & - & 1 & 0,08 \\
\hline & 3572 & 6 & 319 & 6 & 22 & 34 & 3 & 30 & 44 & 2 & 48 & 3 & 1 & 25 & 9 & 3 & 1 & 13 & 25 & 13 & 1 & 1.187 & 100,00 \\
\hline & 256 & 5 & 35 & 2 & 7 & 6 & 3 & 14 & 8 & 2 & 0 & 2 & 1 & 11 & 6 & 2 & 1 & 6 & 5 & 4 & 1 & & \\
\hline
\end{tabular}

$\mathrm{N}^{\circ}$ de espécies de Syrphidae visitantes

(*) espécies abundantes.

Os sirfídeos adultos podem exibir diferenciadas estratégias forrageadoras, normalmente são generalistas, porém há casos onde são considerados especialistas (Haslett 1989; Poctor et al. 1996). Entretanto, neste estudo Syrphus phaeostigma pareceu apresentar uma preferência alimentar por Cinnamomum zeylanicum (Lauraceae); porém, seriam necessários estudos de nicho alimentar para verificar se realmente há uma preferência desta espécie ou se foi apenas a utilização de um recurso abundante naquela época, pois quase todos os indivíduos de S. phaeostigma foram coletados no período de inverno.

A frequiência e abundância das visitas dos sirfídeos nas flores das diferentes famílias de plantas relacionam-se ao tipo (forma e coloração), quantidade e facilidade de acesso aos recursos disponíveis. Logo, flores de colorações escuras, pouco abundantes e de difícil acesso aos recursos florais tendem a apresentar menos visitantes do que as flores de coloração clara (amarela, branca), abundantes e de fácil acesso aos recursos florais.

Verbena bonariensis L. (Verbenaceae) e Eupatorium laevigatum Lam. (Asteraceae) possuem floração abundante, mas apresentam coloração escura (lilás) a qual é pouco atrativa aos sirfídeos, mas comum de se encontrar lepidópteros visitando-as. A maioria das inflorescências de asteráceas apresenta coloração clara (amarela, branca), abundância, e facilidade de acesso aos recursos florais, como em Eryngium horridum (Apiaceae), Ligustrum japonicum (Oleaceae) e Cinnamomum zeylanicum (Lauraceae), as quais estão entre as espécies de plantas com maior número de visitantes.

As inflorescências das espécies de Poaceae são particularmente importantes para disponibilizar pólen aos sirfídeos de pequeno porte (Proctor et al. 1996), o que foi observado com espécies de Allograpta coletadas e com Pseudodoros clavatus. Entretanto, coletaram-se algumas espécies de Palpada visitando espécies desta família.

Neste último caso, as indicações de Proctor et al. (1991) de que a probóscide alongada dos sirfídeos está claramente associada ao hábito forrageador, porém a grande maioria não apresenta adaptações específicas, por se alimentarem geralmente em flores abertas.

As inferências de que a facilidade do acesso aos recursos florais e a coloração clara das flores são os principais responsáveis pela atração dos sirfídeos (Proctor et al. 1996), foram confirmadas através das observações dos sirfídeos 
Tabela III. Famílias e espécies de plantas visitadas, frequiências absoluta e relativa e somatório dos espécimes de Syrphidae visitantes florais, na área do Cinturão Verde de Santa Cruz do Sul, RS, no período de setembro de 2001 a janeiro de 2005.

\begin{tabular}{|c|c|c|c|c|c|c|c|}
\hline Família / Espécie & $\begin{array}{l}\text { Freq. } \\
\text { abso- } \\
\text { luta }\end{array}$ & $\begin{array}{l}\text { Freq. } \\
\text { relativa } \\
{[\%]}\end{array}$ & $\begin{array}{l}\quad \Sigma \text { dos } \\
\text { espécimes } \\
\text { visitantes } \\
\text { por família }\end{array}$ & Família / Espécie & $\begin{array}{l}\text { Freq. } \\
\text { abso- } \\
\text { luta }\end{array}$ & $\begin{array}{l}\text { Freq. } \\
\text { relativa } \\
{[\%]}\end{array}$ & $\begin{array}{l}\Sigma \text { dos } \\
\text { espécimes } \\
\text { visitantes } \\
\text { por família }\end{array}$ \\
\hline Amaranthaceae & & & & & & & \\
\hline Alternanthera sp. & 2 & 0,17 & 4 & sp. 1 & 5 & 0,42 & \\
\hline Celosia argêntea $\mathrm{L}$. & 2 & 0,17 & & sp. 2 & 1 & 0,08 & 30 \\
\hline Anacardiaceae & & & & sp. 3 & 3 & 0,25 & \\
\hline Astronium balansae Engl. & 3 & 0,25 & 3 & Meliaceae & & & \\
\hline Apiaceae & & & & Trichilia claussenii (C. D.C.) & 23 & 1,94 & \\
\hline Eryngium horridum Malme & 569 & 47,94 & 572 & sp. 1 & 21 & 1,77 & 44 \\
\hline Eryngium megapotamicum Malme & 3 & 0,25 & & Nyctaginaceae & & & \\
\hline Asclepiadaceae & & & & Bougainvillaea glabra Choisy & 2 & 0,17 & 2 \\
\hline Asclepia curassavica $\mathrm{L}$. & 6 & 0,51 & 6 & $\begin{array}{l}\text { Oleaceae } \\
\text { Ligustrum japonicum Thunb. }\end{array}$ & 48 & 4,04 & 48 \\
\hline $\begin{array}{l}\text { Asteraceae } \\
\text { Baccharis trimera (Less.) }\end{array}$ & 40 & 3,37 & & Onagraceae & & & \\
\hline Baccharis sp. 1 & 5 & 0,42 & & Ludwigia sp. & 3 & 0,25 & 3 \\
\hline Cosmos bipinnatus Cav. & 94 & 7,92 & & Orchidaceae & & & \\
\hline Elephantopus mollis Kunth & 10 & 0,84 & & Oncidium flexuosum Sims & 1 & 0,08 & 1 \\
\hline Eupatorium laevigatum Lam. & 3 & 0,25 & & Poaceae & & & \\
\hline Senecio brasiliensis (Spreng.) Less & 68 & 5,73 & & Paspalum conspersum Schrad. & 13 & 1,10 & \\
\hline Vernonia florida Gardn. & 11 & 0,93 & 319 & sp. 1 & 7 & 0,59 & \\
\hline Vernonia oxyodonta Malme & 6 & 0,51 & & sp. 2 & 3 & 0,25 & 25 \\
\hline Vernonia polyanthes Less. & 17 & 1,43 & & sp. 3 & 2 & 0,17 & \\
\hline Vernonia puberula Less. & 10 & 0,84 & & Polygonaceae & & & \\
\hline sp. 1 & 8 & 0,67 & & Polygonum punctatum Elliott & 9 & 0,76 & 9 \\
\hline sp. 2 & 29 & 2,44 & & Ranunculaceae & & & \\
\hline sp. 3 & 4 & 0,34 & & Ranunculus muricatus L. & 3 & 0,25 & 3 \\
\hline sp. 4 & 14 & 1,18 & & Rhamnaceae & & & \\
\hline Euphorbiaceae & & & & Hovenia dulcis Thunb. & 1 & 0,08 & 1 \\
\hline $\begin{array}{l}\text { Actinostemon concolor (Spreng.) } \\
\text { Muell. Arg. } \\
\text { Fabaceae }\end{array}$ & 6 & 0,51 & 6 & $\begin{array}{l}\text { Rutaceae } \\
\quad \text { Citrus sinensis (L.) Osbeck } \\
\text { Solanaceae }\end{array}$ & 13 & 1,10 & 13 \\
\hline Bauhinia candicans Benth. & 1 & 0,08 & & Cestrum nocturnum $\mathrm{L}$. & 1 & 0,08 & \\
\hline Desmodium affine Schlecht. & 10 & 0,84 & & Nicotiana tabacum L. & 6 & 0,51 & \\
\hline Lonchocarpus nitidus (Vog.) & 2 & 0,17 & 22 & Solanum acerosum Sendt. & 17 & 1,43 & 25 \\
\hline Mimosa bimucromata (D.C.) O. & & & & sp. 1 & 1 & 0,08 & \\
\hline Kuntze & 9 & 0,76 & & Ulmaceae & & & \\
\hline Lauraceae & & & & Trema micrantha (L.) Blume & 13 & 1,10 & 13 \\
\hline Cinnamomum zeylanicum Blume & 34 & 2,86 & 34 & Verbenaceae & & & \\
\hline Lythraceae & & & & Verbena bonariensis $\mathrm{L}$. & 1 & 0,08 & 1 \\
\hline Heimia sp. & 3 & 0,25 & 3 & & & & \\
\hline $\begin{array}{l}\text { Malvaceae } \\
\quad \text { Sida rhombifolia } \mathrm{L} .\end{array}$ & 21 & 1,77 & & 23 famílias / 51 espécies & 1.187 & 100,00 & 1.187 \\
\hline
\end{tabular}

visitantes em Apiaceae, Asteraceae, Fabaceae, Lauraceae, Malvaceae, Meliaceae, Oleaceae, Poaceae e Solanaceae. Todavia, plantas que possuem recursos florais menos atraentes podem apresentar visitantes mais específicos, isto pode também estar ligado à morfologia das flores, a qual pode promover ou inibir a visita dos insetos, como observado em Verbena bonariensis (Verbenaceae).

A preferência dos sirfídeos pelas temperaturas entre 28 e $32^{\circ} \mathrm{C}$ está intimamente ligada aos períodos de primavera e verão, os quais também possuem a maior disponibilidade de recursos florais.

Estudos anteriores já demonstraram a preferência dos

sirfídeos por horários entre as 10 e 14 horas. Morse (1981) e Gilbert (1985) indicaram picos de atividade no período da manhã e próximos ao meio dia, e Arruda et al. (1998) demonstraram que os picos podem ocorrer antes e depois do meio-dia.

Verificou-se neste estudo que a diversidade da comunidade de Syrphidae é regulada por interações locais entre as espécies, principalmente entre as condições ambientais e disponibilidade de recursos alimentares. As espécies de Apiaceae e Asteraceae são importantes na manutenção da comunidade de Syrphidae, visto que possuem grande quantidade de flores e disponibilidade recursos alimentares abundantes. 
Tabela IV. Freqüências absoluta e relativa dos espécimes de Syrphidae visitantes florais relacionadas à temperatura, no Cinturão Verde de Santa Cruz do Sul, RS, no período de setembro/2001 a janeiro/2005.

\begin{tabular}{ccc}
\hline Temperatura $\left[{ }^{\circ} \mathrm{C}\right]$ & Freq. absoluta & Freq. relativa $[\%]$ \\
\hline $27-27,9$ & 98 & 8,26 \\
$28-28,9$ & 173 & 14,57 \\
$29-29,9$ & 198 & 16,68 \\
$30-30,9$ & 213 & 17,94 \\
$31-31,9$ & 192 & 16,18 \\
$32-32,9$ & 134 & 11,29 \\
$33-33,9$ & 86 & 7,25 \\
$34-34,9$ & 93 & 7,83 \\
\hline Total & 1.187 & 100,00 \\
\hline
\end{tabular}

nos trabalhos de campo e análises estatísticas. A Christian F. Thompson (Smithsonian Institution, EUA), Zuleica de Moraes Borges (Museu Nacional UFRJ), Francisca Carolina do Val (USP), Wilson Sampaio de Azevedo Filho (UFRGS), Tom Reader (University Cambridge - UK), Jean-Louis Hemptinne (ARGE - France), John Pascarella (University of Miami) por fornecerem bibliografia especializada. À Luciane Marinoni (UFPR) pelo acesso a coleção de Syrphidae do DZUP. À Márcia Couri (Museu Nacional UFRJ) pelo empréstimo de material. A Jair Putzke e João Artur Correa Silva (UNISC) pelo auxílio na identificação das espécies de plantas. À Universidade de Santa Cruz do Sul e Universität Tübingen pelo apoio financeiro.

\section{REFERÊNCIAS}

Alves, E. L. \& E. Colischonn. 2001. Cinturão Verde: da preservação a especulação - uma análise ambiental. Laboratório de Geoprocessamento/ Universidade de Santa Cruz do Sul, CD-ROM.

Arruda, V. L. V. de. 1997. Uso de recursos florais por sirfídeos (Diptera: Syrphidae) e interações com outros visitantes. Naturalia 22: 163178.

Arruda, V. L. V. de; M. Sazima \& A. E. Piedrabuena. 1998. Padrões diários de atividade de sirfídeos (Diptera, Syrphidae) em flores. Revista Brasileira de Entomologia 41: 141-150.

Cowell, R. K. 2000. Estimate S: Statistical estimation of species richness and shared species from samples. Version $6.0 \mathrm{~b} 1$. User's Guide and application. Disponível em <http://purl.oclc.org/ estimates>. Acesso em: maio/2003.

Duffield, R. M. 1981. Biology of Microdon fuscipennis (Diptera: Syrphidae) with interpretations of the reproductive strategies of Microdon species found North of Mexico. Proceedings of the Entomological Society of Washington 83: 716-724.

Gilbert, F. S. 1981. The foraging ecology of hoverflies (Diptera, Syrphidae): morphology of the mouthparts in relation to feeding on nectar and pollen in some common urban species. Ecological Entomology 6: 245-262.

Gilbert, F. S. 1985. Ecomorphological relationships in hoverflies (Diptera: Syrphidae). Proceedings of the Royal Society of London 224: 91-105.

Gilbert, F. S.; E. F. Harding; J. M. Line \& I. Perry. 1985. Morphological approaches to community structure in hoverflies (Diptera, Syrphidae). Proceedings of the Royal Society of London 224: $115-130$.

Haslett, J. R. 1989. Interpreting patterns of resource utilization: randomness and selectivity in pollen feeding by adult hoverflies. Oecologia 78: 433-442.

Lobo, E. A. \& G. Leighton. 1986. Estruturas comunitárias de las fitocenosis planctónicas de los sistemas de desembocaduras de ríos y esteros de la zona central de Chile. Revista Biología Marina 22: $1-29$.

Machado, A. de O. \& P. E. A. M. de. Oliveira. 2000. Biologia floral e reprodutiva de Casearia grandiflora Camb. (Flacourtiaceae). Revista Brasileira de Botânica 23: 283-290.

Machado, I. C. \& M. I. Loiola. 2000. Fly pollination and pollinator sharing in two species: Cordia multispicata (Boraginaceae) and Borreria alata (Rubiaceae). Revista Brasileira de Botânica 23: 305-311.

Marinoni, L. \& S. R. Bonatto. 2002. Sazonalidade de três espécies de Syrphidae (Insecta, Diptera) capturadas com armadilha de Malaise no Estado do Paraná, Brasil. Revista Brasileira de Zoologia 19: 95-104.

Marinoni, L.; G. F. G. Miranda \& F. C. Thompson. 2004. Abundância e riqueza de espécies de Syrphidae (Diptera) em áreas de borda e interior de floresta no Parque Estadual de Vila Velha, Ponta Grossa, Paraná, Brasil. Revista Brasileira de Entomologia 48: 554559.

Marinoni, L. \& F. C. Thompson. 2003. Flower flies of southeastern Brazil (Diptera: Syrphidae) Part I. Introduction and new species. Studia Dipterologica 10: 565-578.

Morales, M. N. \& A. Köhler. 2006. Espécies de Syrphidae (Diptera) visitantes das flores de Eryngium horridum (Apiaceae) no Vale do Rio Pardo, RS, Brasil. Iheringia, série Zoologia 96: 41-45.

Morse, D. H. 1981. Interactions among syrphid flies and bumblebees on flowers. Ecology 62: 81-88.

Pascarella, J. B.; K. D. Waddington \& P. R. Neal. 2001. Non-apoid flower-visiting fauna of Everglades National Park, Florida. Biodiversity and Conservation 10: 551-566.

Pérez-Bañon, C.; G. Rotheray; G. Hancock; M. A. Marcos-García \& M. A. Zumbado. 2003. Immature stages and breeding sites of some neotropical saprophagous syrphids (Diptera: Syrphidae). Annals of the Entomological Society of America 96: 458-471.

Proctor, M.; P. Yeo \& A. Lack. 1996. The natural history of pollination. London: Harper Collins Publishers, 479 p.

Ricklefs, R. E. 1996. A economia da natureza. 3. ed. Rio de Janeiro: Guanabara Koogan, 470 p.

Rotheray, G. E.; Hancock, E. G. \& Marcos-García, M. A. 2007. Neotropical Copestylum (Diptera, Syrphidae) breeding in bromeliads (Bromeliaceae) including 22 new species. Zoological Journal of the Linnean Society 150: $267-317$.

Souza-Silva, M.; J. C. R Fontenelle \& R. P. Martins. 2001. Seasonal abundance and species composition of flower-visiting flies. Neotropical Entomology 30: 351-359.

Thompson, F. C. 1981. The Flower Flies of the West Indies (Diptera: Syrphidae). Memoirs of the Entomological Society of Washington 9: 1-200

Thompson, F. C. 1999. A key to the genera f the flower flies (Diptera: Syrphidae) of the neotropical region including descriptions of new genera and species and a glossary of taxonomic terms. Contributions on Entomology International 3: 319-378.

Thompson, F. C. 2003. Austalis, a new genus of flower flies (Diptera: Syrphidae) with revisionary notes on related genera. Zootaxa 246: $1-19$.

Thompson, F. C. (editor) 2005. Biosystematic Database of World Diptera. Version 7.5. Available from http://www.sel.barc.usda.gov/ names (accessed 11 Sep 2006).

Thompson, F. C.; J. R. Vockeroth \& Y. S. Sedman. 1976. Family Syrphidae. A catalogue of the Diptera of the Americas south of the United States, Departamento de Zoologia, Secretaria de Agricultura., v. 46, 195 p.

Vockeroth, J. R. \& F. C. Thompson. 1987. Syrphidae. Manual of Nearctic Diptera, Agriculture Canada, Research Branch Monograph 2: 713-743. 